Previous work has considered a relation of offspring sex ratio with time to pregnancy. ${ }^{5}$ A slightly reduced sex ratio was found in infertile couples (time to pregnancy $>1$ year). Comparison with the current findings, however, is complicated by the fact that no adjustments or subdivisions were made for assisted reproduction and the prevalence of reported infertility in the study population was unusually high (18\%).

Contributors: LS had the idea for the study, analysed the statistics, and wrote the final manuscript. RdeB wrote the study protocol and got the financial support. LS, RdeB, PvandenB, and GE developed the questionnaires, discussed the statistical analyses, and discussed and revised the manuscript. LS is guarantor.
Funding: Dutch Health Council.

Competing interests: None declared.

Ethical approval: University Hospital Maastricht.

1 Ericsson RJ, Langevin CN, Nishino M. Isolation of fractions rich in human Y sperm. Nature 1973;246:421-4.

Keye WR, Chang RJ, Rebar RW, Soules MR, eds. Infertility; evaluation and treatment. Philadelphia: WB Saunders, 1995.

Martin JF. Birth order, coital rates, polygyny, stress, and the secondary sex ratio: a reply to James. Soc Biol 1997;44:283-8.

Martin JF. Length of the follicular phase, time of insemination, coital rate and the sex of offspring. Hum Reprod 1997;12:611-6.

Weijin Z, Olsen J. Offspring sex ratio as an indicator of reproductive hazards. Occup Environm Med 1996;53:503-4.

(Accepted 5 September 2005)

\title{
Family history of breast cancer and cost of life assurance: a test case comparison of current UK industry practice
}

\author{
A Hunter, S E Humphries
}

London IDEAS Genetics

Knowledge Park,

Institute of Child

Health, London

WC1N 1EH

A Hunter

genetics knowledge

park manager

Centre for

Cardiovascular

Genetics, BHF

Laboratories,

London WC1E 6JF

S E Humphries

professor of

cardiovascular

genetics

Correspondence to:

A Hunter

ahunter@

BMJ 2005;331:1438-9
Under the current recently extended moratorium, applicants for life assurance need not disclose the results of predictive genetic tests. ${ }^{1}$ The exception is for policies exceeding certain values, when insurers may seek results of tests approved by the government's Genetics and Insurance Committee. The committee expects to receive applications for the use of adverse results from tests for the BRCA1 and BRCA2 genes. Currently, insurers may, and often do, seek family histories. Substantial epidemiological data describe the relative risks of developing breast cancer depending on family history and age. ${ }^{2}$ Preventive action for women at risk can include early enrolment on surveillance programmes and prophylactic surgery. Evidence is emerging for the benefits of the former, ${ }^{3}$ and evidence is strong for risk reduction by the latter. ${ }^{45}$

\section{Participants, methods, and results}

We surveyed 21 companies representing $100 \%$ of the reinsurance market and $68 \%$ of the life and pensions market in the United Kingdom. We asked the companies to assess a fictional proposal for a 20 year policy (paying benefit only on death) by applying an excess mortality rating, defined as the percentage increase over the assumed rate of mortality. The applicant (scenario 1) was a 35 year old woman with unremarkable personal and family histories, except for breast cancer in the mother diagnosed at age 35. In line with standard insurance application forms, only first degree family history was given. In scenario 2 , the same applicant had enrolled on a mammographic surveillance programme with no adverse results reported. In scenario 3, the same applicant had undergone prophylactic double mastectomy and oophorectomy (figure).

Sixteen companies responded. The responding and non-responding groups included a similar variety of company profiles (for example, global operations and size). Nine would not increase premiums under any of the scenarios. Six would increase premiums under scenario 1 (rating $+50 \%$ or $+75 \%$ ), and of these, four would not load under scenario 3 , and two would

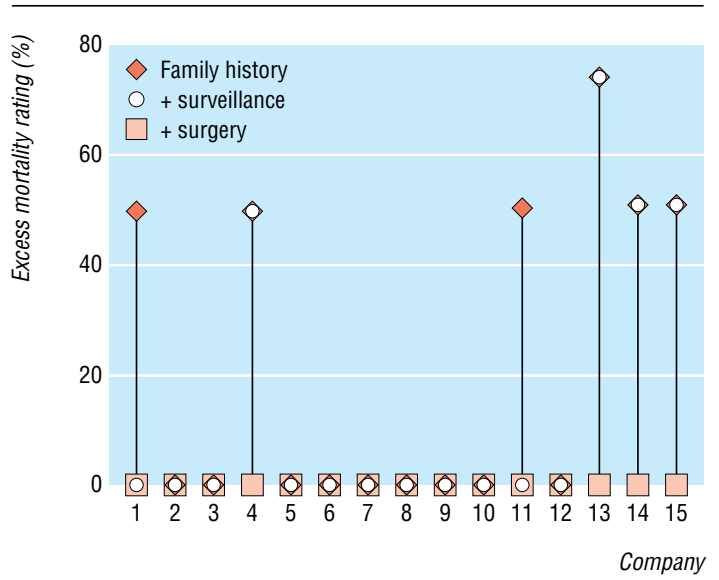

Percentage excess mortality ratings applied by life insurance and reinsurance companies in 2004-5. All companies gave one rating for the full product term, except for one that did not specify the rating or duration and is not shown

not load under either scenario 2 or 3 . One small UK company would not raise premiums under scenario 1 or 2, but would apply an unspecified increase under scenario 3 .

\section{Comment}

Most life insurance companies that responded to the survey would offer a standard premium in scenario 1 (unremarkable except breast cancer in the mother diagnosed at age 35). The lifetime relative risk of developing breast cancer for the applicant in scenario 1 is 5.7 (2.7 to 11.8). ${ }^{2}$ Using life tables developed at the Cambridge Genetics Knowledge Park (A Butterworth, personal communication, 2005) this translates into a 20 year breast cancer mortality risk (from age 35 ) of $1.95 \%$, compared with $0.6 \%$ for the general population - that is, three times higher.

It is reassuring that not only did all companies that rated under scenario 1 remove the rating after prophy- 
lactic surgery, but that two companies also gave credit for enrolment on a mammographic surveillance programme. Another company reported that this will be part of a review of policy, and in future it may also credit mammographic surveillance. The variation in the industry's responses show that women with a family history who are offered a high rating by one company may receive a lower rating if they approach a second company.

No respondent said that they would request further family history to see if second degree relatives were affected. Disclosure of more cases of cancer in second degree relatives could considerably increase the assessed risk. The artificial nature of the survey might have implied, however, that no further family history existed to be disclosed, so we cannot comment on whether second degree relative information would be requested in reality. How access to adverse test results for BRCA1 or BRCA2 would influence the response to a similar survey in the future remains to be seen.

We thank the life insurance companies for participating in the study, and Adam Butterworth, Cambridge Genetics Knowledge Park, and Alan Tyler, independent insurance and health consultant, for advice and review of the manuscript.

Contributors: AH designed the study with advice from $\mathrm{SEH}$, and did the survey. AH wrote the paper. Both authors critically revised the paper. $\mathrm{SEH}$ is guarantor.

Funding: London IDEAS Genetics Knowledge Park grant, funded by the Departments of Health, and Trade and Industry. Competing interests: None declared.

Ethics approval: Not needed.

\section{What is already known on this topic}

Insurers currently do not use adverse predictive test results for BRCA1 and BRCA2 genes, but this could change and could affect an increasing number of women because of the National Institute for Health and Clinical Excellence's 2004 guidelines recommending genetic testing for certain women at high risk

Applicants must disclose family history to inform risk assessment, but they can also disclose other relevant medical information, such as preventive surveillance or surgery that they may have taken

\section{What this study adds}

Reassuringly, the UK insurance industry currently makes an appropriate assessment of women with a family history of breast cancer, including how preventive action affects risk

Department of Health, Association of British Insurers. Concordat and moratorium on genetics and insurance. London: Department of Health, 2005.

2 Collaborative Group on Hormonal Factors in Breast Cancer. Familial breast cancer: collaborative reanalysis of individual data from 52 epidemiological studies including 58,209 women with breast cancer and 101,986 women without the disease. Lancet 2001;358::1389-99.

3 Tabar L, Yen MF, Vitak B, Chen HH, Smith RA, Duffy SW. Mammography service screening and mortality in breast cancer patients: 20-year follow up before and after introduction of screening. Lancet 2003:361:1405-10.

4 National Institute for Clinical Excellence. Clinical guidelines for the classification and care of women at risk of familial breast cancer. London: NICE 2004.

Rebbeck TR, Friebel T, Lynch HT, Neuhausen SL, van’t Veer L, Garber JE, et al. Bilateral prophylactic mastectomy reduces breast cancer risk in BRCA1 and BRCA2 mutation carriers: the PROSE study group. J Clin Onc 2004;22:1055-62

(Accepted 8 November 2005)

\title{
Severe placental malaria and maternal shortness, thinness, and small skeletal size in rural Congo: cohort study
}

\author{
Hermione J Lovel, Rachel M Newby, Valerie F Hillier
}

Despite global partnerships that aim to eradicate malaria, few studies have investigated susceptibility to severe placental malaria infection (apart from in primigravida ${ }^{1}$ ). Reduced fetal growth ${ }^{1}$ and maternal anaemia sequelae ${ }^{2}$ are known, however, to have serious consequences. We measured mothers at antenatal booking and later explored severe placental malaria as part of a study on factors affecting fetal growth.

\section{Participants, methods, and results}

We investigated a cohort of 436 consecutive rural Bantu women with a singleton pregnancy, with a gestation of less than 24 weeks at booking (determined by ultrasonography), and planning to deliver in Kasaji Hospital in this remote but settled rural area of Katanga province in the Democratic Republic of Congo. A third $(31 \%, 135)$ were primigravida. ${ }^{3}$ RMN followed them all through pregnancy to delivery, between 1996 and 1998 (unaware of their placental malaria status at this stage). RMN took placental impression smears using a rigorous protocol. HC later examined them and counted parasites per field (without antenatal measurements) at the Tropical Diagnostic Laboratory (Dublin). RMN graded parasite density $(0=$ no parasites in 150 fields; $1=1-49$ parasites per 50 fields; $2=$ more than 50 parasites per 50 fields). Because supplies were limited, women were not given chemoprophylaxis for malaria, but prompt treatment with chloroquine was available for symptoms. We found placental infection in 322 women (74\%; 125 (93\%) primigravida, 197 (65\%) multigravida; and 85 (58\%) gravida six or more), mainly Plasmodium falciparum.

Women later found to have severe (grade 2) placental malaria were shorter and thinner at booking than those with no placental malaria (table 1; statistically significant decreasing gradient with placental malaria severity groups; analysis of variance $\mathrm{P}<0.05$ ), for three measures of skeletal smallness (height, interiliac crests, and intergreater trochanters) and three measures of thinness (body mass index, midarm circumference, and weight). Foot size and triceps skinfold thickness showed similar but non-significant trends.
WHO Collaborating

Care, School of

Primary Care,

University of

Manchester,

Rusholme Health

Centre, Manchester

M14 5NP

Hermione J Lovel senior lecturer

Katanga Province,

DR Congo Kasaji

Hospital

Rachel M Newby

midwife

Division of Imaging

Science and

Biomedical

Engineering,

University of

Manchester,

Manchester M13 9PL

Valerie F Hillier

senior lecturer

Correspondence to: H J Lovel hlovel04@ yahoo.co.uk

BMJ 2005;331:1439-40 